\title{
EUROPEAN CENTRAL BANK
}

\section{WORKING PAPER SERIES}

NO 905 / JUNE 2008

\section{A PERSISTENCE-WEIGHTED}

\section{MEASURE OF CORE}

\section{INFLATION IN}

THE EURO AREA

by Laurent Bilke and Livio Stracca

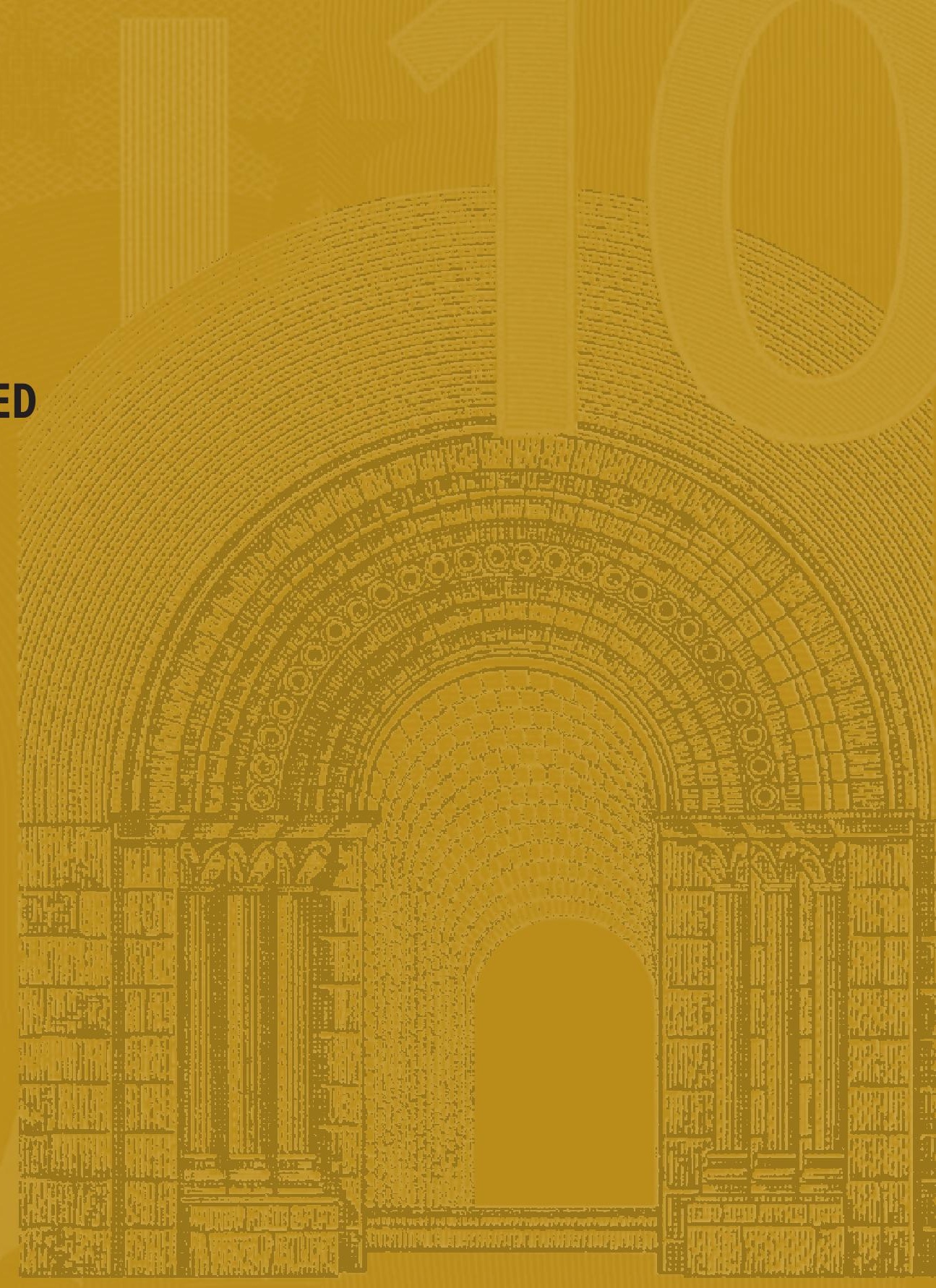




\section{A PERSISTENCE-WEIGHTED MEASURE OF CORE INFLATION IN THE EURO AREA'}

by Laurent Bilke ${ }^{2}$ and Livio Stracca ${ }^{3}$

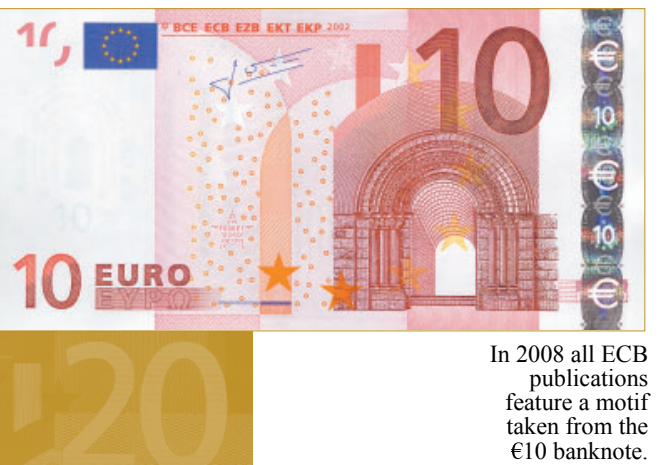

This paper can be downloaded without charge from http://www.ecb.europa.eu or from the Social Science Research Network electronic library at http://ssrn.com/abstract_id=1138598.

I The views expressed in this paper are only those of the author and are not necessarily shared by the European Central Bank. We thank a referee for constructive comments which materially improved the paper. 2 Lehman Brothers, 25 Bank Street, London EI4 5LE, United Kingdom; e-mail: laurent.bilke@lehman.com

This paper was mostly written when I was at the European Central Bank, Euro Area Macroeconomic Developments Division. 


\section{(C) European Central Bank, 2008}

\section{Address}

Kaiserstrasse 29

60311 Frankfurt am Main, Germany

\section{Postal address}

Postfach 160319

60066 Frankfurt am Main, Germany

\section{Telephone}

+496913440

Website

http://www.ecb.europa.eu

Fax

+496913446000

\section{All rights reserved.}

Any reproduction, publication and reprint in the form of a different publication, whether printed or produced electronically, in whole or in part, is permitted only with the explicit written authorisation of the ECB or the author(s).

The views expressed in this paper do not necessarily reflect those of the European Central Bank

The statement of purpose for the ECB Working Paper Series is available from the ECB website, http://www.ecb.europa. eu/pub/scientific/wps/date/html/index. en.html 


\section{CONTENTS}

Abstract

Non-technical summary

1 Introduction

2 Measures of inflation persistence

3 The computation of persistence-weighted measures of core inflation in the euro area

3.1 The data

3.2 Computation

4 Results

5 A closer look at the individual HICP components

5.1 The weight of the complete set of 93 components

5.2 What are the most persistent components of the HICP? Some surprises

6 Conclusions

References

European Central Bank Working Paper Series 


\begin{abstract}
We propose a new core inflation measure for the Euro area which places the emphasis on the more lasting, i.e. persistent, price developments at a disaggregated level. The importance of each component of the HICP is reweighted according to its relative persistence, as measured by the sum of the autoregressive coefficients or by an indicator of mean reversion. Unlike headline inflation, our baseline core inflation measure is highly correlated with ECB monetary policy decisions, which could mean that it contains ex ante (pre monetary policy) information on inflationary pressure.
\end{abstract}

Keywords: core inflation, inflation persistence.

JEL codes: E31. 


\section{Non-technical summary}

Central banks typically define their inflation objective in reference to a medium or long term horizon. The ECB for instance is committed to maintain price stability defined as a year on year increase in the Euro area Harmonised Index of Consumer Prices (HICP) of below 2\% over the medium term. In the literature, one of the possible ways to disentangle noise from the more lasting developments is to refer to a core inflation measure that would exclude the most erratic or volatile components. Indeed, most statistical institutes have built various core inflation measures by excluding a fixed list of presumed volatile items, such as food and energy in the United States or unprocessed food and energy in Europe. Some central banks have also chosen to monitor particularly closely such measures, like the Bank of Canada. Instead of permanently excluding the presumed most volatile components, it has also been proposed to measure the variance of each component at each period of time and then use this information to re-weigh the basket by giving less weight to the more volatile components.

In this paper we take a somewhat different approach and build a measure of core inflation excluding or giving less weight to the less persistent processes. Blinder (1997) first made very clearly the case that this view differs from the first approach and that it may be closer to the question policy-makers typically have in mind, namely: "what part of each monthly observation on inflation is durable and what part is fleeting?".

In particular, in this paper we build three alternative persistence-weighted measures of core inflation in the euro area and we analyse their properties in terms of correlation with current and future headline HICP inflation and with the ECB monetary policy decisions. In fact, in an environment of price stability in which the central bank reacts aggressively and in a timely manner to inflationary pressure it is highly unlikely to find core inflation (or any other) indicators that may be systematically correlated with future headline inflation. More revealing about the potential usefulness of a core inflation indicator should be its correlation with the monetary policy decisions. We show that our baseline measure of persistence-weighted core inflation scores remarkably well in this respect. However, it goes without saying that, given the wide information set taken into consideration by the $\mathrm{ECB}$, it is highly unlikely that any core inflation indicator can be a "sufficient statistic" to explain monetary policy decisions in the euro area.

A further interesting result of this study is that there appears to be significant time variation in the persistence of the sub-components of the euro area HICP. In contrast with the findings of the Inflation Persistence Network, we find services prices to be under-weighted in our persistence-weighted indicator, while energy and food prices are over-weighted compared with the original HICP weights. This reflects, in our view, the different nature of the shocks which have predominantly hit prices in the sample period we use in this paper (from 1995 onwards) compared with the longer sample period studied in previous work. 


\section{Introduction}

Central banks usually define their inflation objective in reference to a medium or long term horizon. The ECB for instance is committed to maintain price stability defined as a year on year increase in the Euro area Harmonised Index of Consumer Prices (HICP) of below 2\% over the medium term. Thus, its economic analysis has to disentangle the short-term shocks from the more lasting developments. In the case of the ECB, this is addressed through a broad economic and monetary analysis which allows for a cross-checking of the economic trends.

One of the possible ways to disentangle noise from the more lasting developments is to refer to a core inflation measure that would exclude the most erratic or volatile components. ${ }^{1}$ Most statistical institutes have built various core inflation measures by excluding a fixed list of presumed volatile items, such as food and energy in the United States or unprocessed food and energy in Europe. Some central banks have also chosen to monitor particularly closely such measures, like the Bank of Canada. Instead of permanently excluding the presumed most volatile components, it has also been proposed to measure the variance of each component at each period of time and then use this information to re-weigh the basket by giving less weight to the more volatile components.

A somewhat different approach is to state that what should rather be considered as noise are the high frequency movements. Monetary policy, given his medium term perspective, should put more emphasis on the more lasting movements and disregard the shocks that are likely to be soon reverted. Putting this idea into practice, this would imply excluding or giving less weight to the less persistent processes. Blinder (1997) first made very clearly the case that this view differs from the first approach and that it may be closer to the question policy-makers typically have in mind, namely: "what part of each monthly observation on inflation is durable and what part is fleeting?".

It is easy to show that the two approaches can lead to substantially different results. For instance, the higher the root $\rho$ of a first order autoregressive process, the higher its overall variance $\gamma\left(\gamma=\sigma^{2} /\left(1-\rho^{2}\right), \sigma^{2}\right.$ being the variance of the perturbation). Therefore, an item can be excluded from the core inflation measure (or given less weight) on the basis of the first approach (large variance), but be included on the basis of the second approach (as it exhibits high persistence). As an illustration, Figure 1 below shows two series which have the same variance of the perturbation $\left(\sigma^{2}=1\right)$ but a different persistence (their $\mathrm{AR}(1)$ roots are respectively 0.2 and 0.9 ). On the basis of the first approach, one would exclude from the core inflation measure the second series (with $\rho=0.9$ and $\gamma=5$ ), while on the basis of the second approach, one would exclude the first one $(\rho=0.2$ and $\gamma=1)$. Following Blinder's suggestion, our intuition is that the second time series, more persistent and more volatile, has a richer information content that the first one for monetary policy.

\footnotetext{
${ }^{1}$ There are several useful surveys on core inflation indicators and their usefulness for monetary policy; see, for example, Mankikar and Paisley (2004), Roger (1998), and Wynne (1999). For the specific case of the euro area, see ECB (2001) and Vega and Wynne (2003).
} 


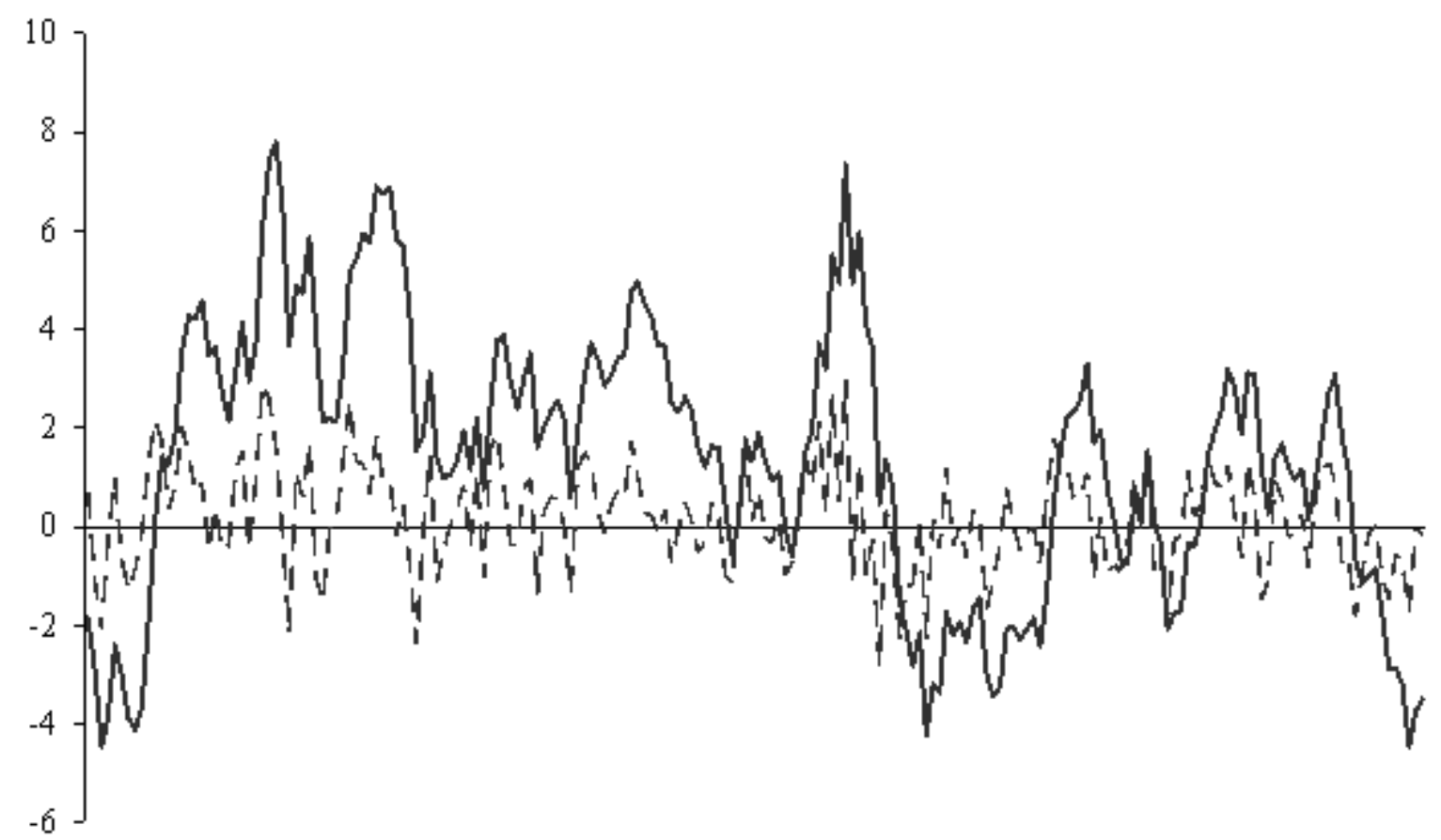

Figure 1: Simulated series: dotted line with $\rho=0.2, \gamma=1$; solid line with $\rho=0.9, \gamma=5$.

In the context of the Eurosystem Inflation Persistence Network ${ }^{2}$, empirical studies have however revealed that energy and unprocessed food prices which are usually excluded from the core measures as they are presumed to exhibit an elevated variance are also typically the less persistent components of the basket (Bilke 2005 and Altissimo et. al. 2006). Therefore both approaches could justify the exclusion of the prices of these two categories of goods from the core inflation measure. This is presumably because the idiosyncratic part of the variance of the perturbation for these prices is larger than for the other items. However, the general case remains valid that using variance as the exclusion criterion does not necessarily imply excluding the less persistent price changes.

This motivates the implementation of a core inflation measure directly based on the persistence criterion, the less persistent items being excluded or given less weight than the others. The first attempt to operationalise Blinder's concept was made by Cutler (2001) who proposed such a price index for the United Kingdom. A similar attempt was made for Malta by Demarco (2004). In this paper, we propose such a measure for the euro area.

One often problematic issue related to core inflation measures is to the criteria along which they can be appraised. As previously stated, core inflation measures are certainly

\footnotetext{
${ }^{2}$ See ECB (2005) for additional information on the Eurosystem Inflation Persistence Network and its results.
} 
primarily meant to remove some noise from headline inflation and this can imply that they should be able to track possible trends in headline inflation (Marques, Neves and Sarmento 2003 propose some statistical criteria to asses this property). However, it can be argued that the ability to track a trend in headline inflation or the out of sample forecast performance is not necessarily the proper way to assess the usefulness of core inflation measures. If such a measure really had a significant information content for future inflation, one can expect that the central bank would include such information in its reaction function and, as a consequence, realized inflation would deviate from the presumably leading core inflation signal (Mankikar and Paisley, 2004). Therefore, we suggest to compare core inflation measures with monetary policy decisions. If a certain core inflation measure captures impending and sustained swings to inflationary pressures and the central bank reacts to such pressures, then the core inflation indicator will be correlated with the monetary policy instrument, rather than with future headline inflation.

In addition, we propose some new features in the implementation of the persistence based core inflation measure. Cutler (2001) and Demarco (2004) have used an AR(1) process to assess the persistence of each component, but the inflation persistence literature has also referred to other measures of persistence. Therefore, we compare different measures of core inflation based on different ways to evaluate persistence. In order to avoid over-estimating persistence, it is also necessary to control for possible breaks in the mean of the processes, which we include in our framework. Last, our core inflation measure can be considered as a real time measure, in the sense that the persistence weight of each price sub-index is assessed only with the available information at a given point in time.

The paper is organised as follows. In Section 2 we present some measures of inflation persistence and select those measures which we use in the empirical analysis. Section 3 describes the actual computation of the persistence-weighted core inflation index, and the results are reported and commented on in Section 4. Section 5 analysis the correlation of different core inflation measures, including the persistence-weighted ones proposed in this paper, with euro area headline inflation. Section 6 concludes.

\section{Measures of inflation persistence}

Inflation persistence relates to the speed with which inflation converges to equilibrium (or baseline) after a shock: the more time this return takes, the more persistent the process. As argued by Andrews and Chen (1994), the long-term persistence properties of time series are captured by their impulse response function (IRF). However, the IRF being an infinite vector of numbers, one rather needs in practice to refer to a scalar measure of persistence. The most common one, proposed by Andrews and Chen (1994) and used in most of the literature on inflation persistence ${ }^{3}$, is the sum of the autoregressive coefficients which relates

\footnotetext{
${ }^{3}$ For instance, the literature that has emerged in the context of the Eurosystem Inflation Persistence Network (IPN). See Altissimo et al. (2006) for a summary of the macroeconomic side of this project.
} 
to the Cumulative Impulse Response (CIR).

There are other measures also based on an auto-regressive process, like the half-life or the largest autoregressive root. However, those measures are less widely used, at least in the literature measuring inflation persistence. The half life can be somewhat misleading when the IRF is not continuously decreasing with time, whereas the largest autoregressive root can provide a misleading signal depending on the presence or not of a long suite of AR parameters.

Dias and Robalo Marques (2005) proposed another interesting measure based on a different concept: a process is persistent when it does not cross its mean very often. Therefore, the frequency of mean reversion can be directly taken as an indicator of persistence. We propose to try both measures - the sum of the autoregressive coefficients and the mean reversion - as the indicators of persistence.

Concerning the sum of the autoregressive coefficients (SAR), which will be considered as the baseline measure in the continuation of this paper, for practical reasons we will not implement the procedure proposed by Andrews and Chen (1994) aimed at dealing with a downward bias to the autoregressive coefficients when their value is close to zero in finite sample. We are conscious that this choice can theoretically slightly distort on one side the distribution of persistence. However, given the relatively low number of highly persistent processes this should have a very limited impact on our measure. Cutler (2001) and Demarco (2004) have used an AR(1) to assess the persistence of each component; instead, we do not impose any predetermined order to our AR processes but rather use an information criterion to select it.

In addition, we propose a third measure based on the leading properties of each subcomponent: the structure of the basket will be transformed in order to give more weight to the items which are better leading indicators of overall HICP inflation developments. This measure represents the direct implementation of one of the ideas put forward by Blinder (1997): the more informative components of inflation are those which are most correlated with headline inflation some time ahead, i.e. at an horizon that may be relevant for monetary policy.

\section{The computation of persistence-weighted measures of core inflation in the euro area}

\subsection{The data}

The data used in this study cover the 93 components of the Harmonised Index of Consumer Prices in the euro area, as computed by Eurostat according to the COICOP classification. ${ }^{4}$

\footnotetext{
${ }^{4}$ The Classification of Individual Consumption by Purpose (COICOP) is a Reference Classification published by the United Nations Statistics Division that divides the purpose of individual consumption expenditures incurred by three institutional sectors, namely households, non-profit institutions serving households
} 
The data span from January 1995 to January 2007, i.e. covering over twelve years of monthly data. A handful of components are not reported as from 1995 and start at a later date, due either to unavailability of back data or to the introduction of new items in the HICP. These components are dealt with based on an automatic procedure described in the next subsection. The data we use are seasonally adjusted using the X12 ARIMA procedure ${ }^{5}$; but using seasonally unadjusted data leads to very similar results. Moreover, although the focus of the analysis is on real-time computation, we use data revised ex post; the revisions to the HICP data are, however, rather small and this should not have a significant impact on our results.

The table below reports the main groupings of the individual HICP components together with their HICP (COICOP) weight in $2006 .^{6}$

Table 1 - Main items in the HICP

\begin{tabular}{||c||l|}
\hline \hline Item & COICOP weight in 2006 \\
\hline \hline Food (incl. alcohol and tobacco) & 19.3 \\
\hline o/w processed & 7.4 \\
\hline o/w unprocessed & 11.8 \\
\hline Industrial goods & 39.9 \\
\hline o/w non-energy & 30.7 \\
\hline o/w energy & 9.2 \\
\hline Services & 40.8 \\
\hline o/w rents paid by tenants & 6.3 \\
\hline o/w restaurants and cafes & 6.8 \\
\hline o/w housing & 10.3 \\
\hline o/w transport & 6.4 \\
\hline
\end{tabular}

Before turning to measuring persistence for each component, an intermediate step has to be considered. As previously stated, our two measures of persistence aim at gauging the speed of return to a baseline (mostly a mean in practice) after a shock. It is well known in the literature that the persistence of a process will be overestimated if one ignores that a discrete break in the mean occurred at some point in time: one would take for a slow return to baseline what in fact is a structural (permanent) change (Perron 1990). Altissimo et. al. (2006) for instance have shown that the unit root can hardly be excluded over long period for inflation if the structural breaks are ignored, while inflation persistence appears to be relatively low once the discrete changes in the mean are incorporated.

Therefore, we have tested whether structural breaks could be detected in the month-onmonth rates of changes of each individual price series. As in Bilke (2005) and Corvoisier and

and general government. The COICOP classification is also used by Eurostat.

${ }^{5}$ Note that the procedure is run on the ex post data, i.e. not in real time.

${ }^{6}$ The HICP weights are changed once every year. 
Mojon (2005), we have done so using the Altissimo and Corradi (2003) procedure, which is an improved version of the standard Bai and Perron test for multiple breaks. Our findings are unambiguous: none of the series exhibits at least one structural break in the mean over the period studied. ${ }^{7}$ This result is consistent with the findings of the Eurosystem Inflation Persistence Network, for which usual break dates in the euro area are early 1970s, the mid 1980s and the early 1990s (Altissimo et. al. 2006), hence out of our estimation period.

\subsection{Computation}

We now turn to describe the actual computation of the three persistence-weighted core inflation measures. ${ }^{8}$ The one-month growth rates of the 93 individual components of the HICP are computed and de-meaned. The computation of the core inflation series starts in January 1998. In order to control for missing values not only at the start of the period but also in previous years (given that the estimation of measures of persistence requires some back data) we exclude from the computation those components that, at each point in time $T$, present missing values up to 2 years before $T$.

Let $p_{i t}$ be the log of the i-th component of the HICP at time t, and let $\Delta p_{i t}$ be its first difference (i.e. the one-month growth rate of the i-th component). For the first measure of persistence based on the sum of the autoregressive coefficients, for each component we run the following regression recursively between January 1998 and time $T$, i.e. only using data available in real time ${ }^{9}$

$$
\Delta p_{i t}=c+\sum_{j=1}^{q_{i}} \alpha_{j}^{i} \Delta p_{i, t-j}+\varepsilon_{i t}
$$

where the order $q_{i}$ of the regression is chosen so as to minimise the Schwarz information criterion, i.e. the one that is typically selected when obtaining a parsimonious model is considered to be of importance. The search over the $q_{i}$ parameter which minimises the Schwarz criterion is conducted for values between 1 and 12 , the latter included in order to cater for possible residual seasonality in the price series (though the series we use are seasonally adjusted). For most components, however, the $q_{i}$ is a very small number and equation contain no more than one or two lags, which makes the equation quite reasonable in terms of degrees of freedom already in the early part of the sample period.

The the first persistence measure, which we take as our baseline measure in the continuation of the paper, is simply

$$
\widetilde{\gamma}_{i T}^{A R}=\sum_{j=1}^{q_{i}} \alpha_{j}^{i}
$$

\footnotetext{
${ }^{7}$ This result appears to be relatively robust as it is left unchanged by several parameterisation of the bandwidth used for the computation of HAC variance (see Bilke 2005 for more details on this issue). The critical value of the test has been adjusted for the sample size, as suggested by Altissimo and Corradi (2003).

${ }^{8}$ The computation is done in Eviews; the program is available from the authors upon request.

${ }^{9} \mathrm{We}$ also tried to include a deterministic time trend in the regression, but it does not make any difference in the final result.
} 
as estimated up to time $T$. Should any $\widetilde{\gamma}_{i T}^{A R}$ be negative, we put the measure at zero, as in Cutler (2001). Obviously, the $\widetilde{\gamma}_{i T}^{A R}$ are then re-scaled for them to sum up to one at each time $T$, as we do with the other two variants that we consider.

There is little to say on the computation of the second measure based on the number of times a variable crosses its mean, in this case zero since the variables are de-meaned; we will refer to this second measure as $\widetilde{\gamma}_{i T}^{M C}$, where MC stays for "mean crossing". Turning to the third measure based on the correlation with headline annual HICP inflation one year ahead, the computation runs as follows. At each time $T$, we compute the correlation between headline HICP inflation and the annual increase in each component of the HICP, lagged one year (since data after $T$ are not available in a real time computation). Also for this measure the persistence weight is set to zero if the correlation turns out to be negative. We will refer to the weights identified by this third measure as $\widetilde{\gamma}_{i T}^{\text {Corr }}$.

In the same way as the HICP, the index numbers for the persistence-weighted indicators are reconstructed using a chain-linked computation (i.e. based on the cumulated growth rate from the level of the index in December of the previous year). Thus, the results are fully comparable with the headline HICP.

It should be added at this stage that the computation of the persistence-weighted index might be problematic especially in the first part of the sample period, due to the lack of a long time price series for the euro area. In order to check whether the small sample bias may be a significant problem in practice, we recomputed the baseline persistence-weighted core inflation measure using the final ex post weights as well as weights computed from regressions on a 4-year rolling window. The results for these variants were very close to the original calculation, suggesting that the uncertainties related to the small sample may not be economically very significant. ${ }^{10}$

\section{Results}

The baseline persistence-weighted core inflation measure and the two proposed variants are computed for the sample period from January 1998 to January 2007. The result for the baseline measure (taking the annual growth rate for illustration purposes) is reported in Figure 2 below. In a simple visual inspection, three features stand out. First, the behaviour of the series is interestingly different from, and somewhat more volatile than, headline HICP inflation, which has remained (rather un-interestingly) in a relatively narrow margin since 2000. Second, unlike other popular core inflation measures (more on this later), the persistence-weighted index is a coincident if not slightly leading indicator of headline inflation, and not a lagging one. For example, HICP inflation excluding energy and unprocessed food is a clearly lagging indicator of headline inflation (see Figure 3). Third, and most notably, there is a striking correlation between the behaviour of the index and the ECB monetary policy stance as measured by its main refinancing rate (see Figure 4).

${ }^{10}$ The calculation is not reported for brevity but is available from the authors upon request. 


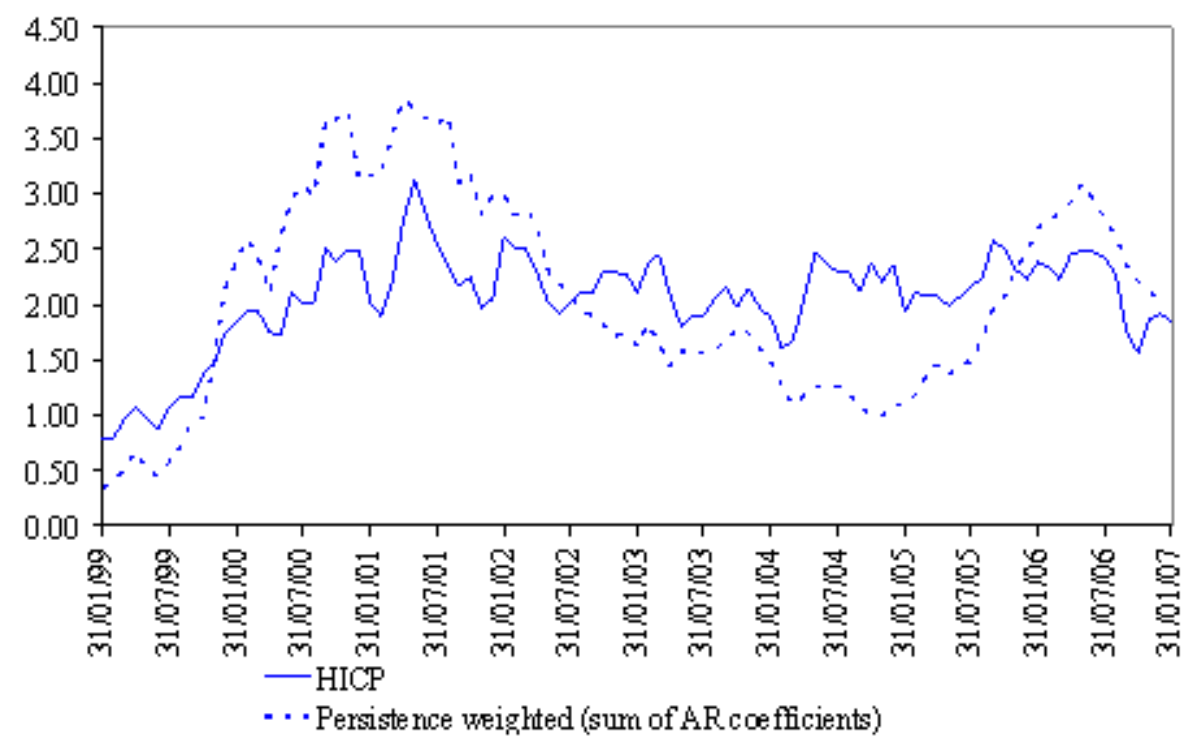

Figure 2: Headline inflation and persistence weighted (sum of AR) core inflation.

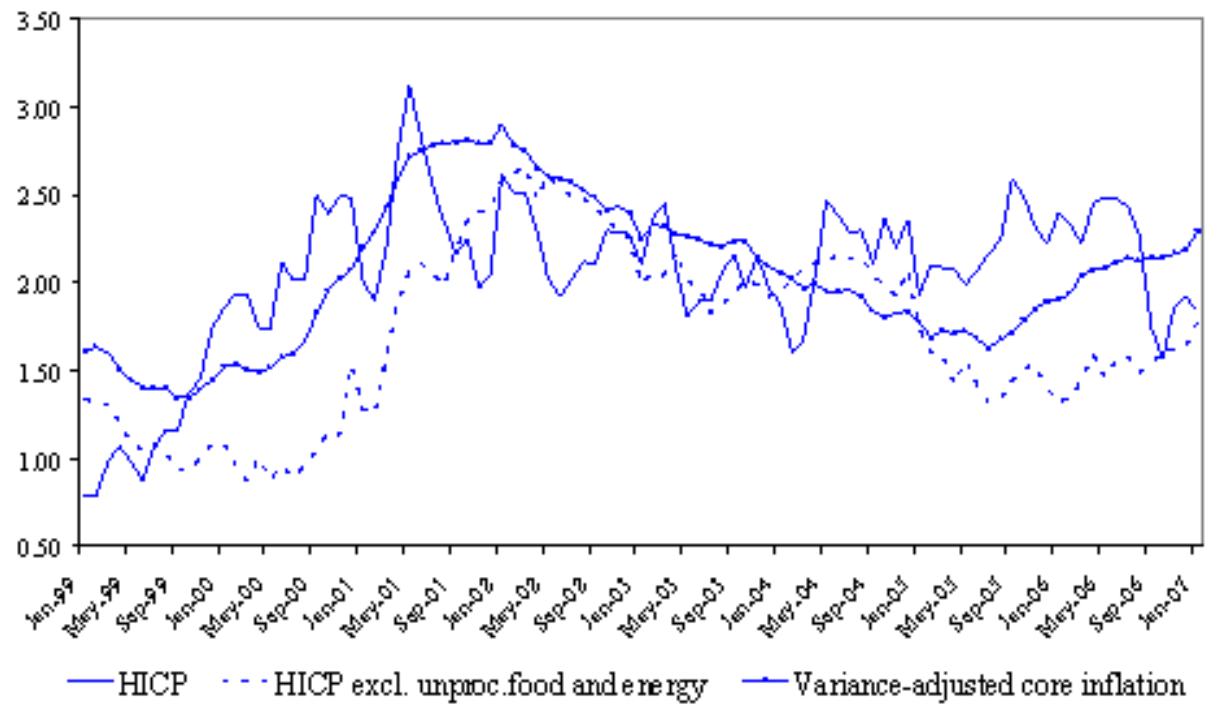

Figure 3: Headline inflation, variance-adjusted core inflation and inflation excluding unprocessed food and energy. 


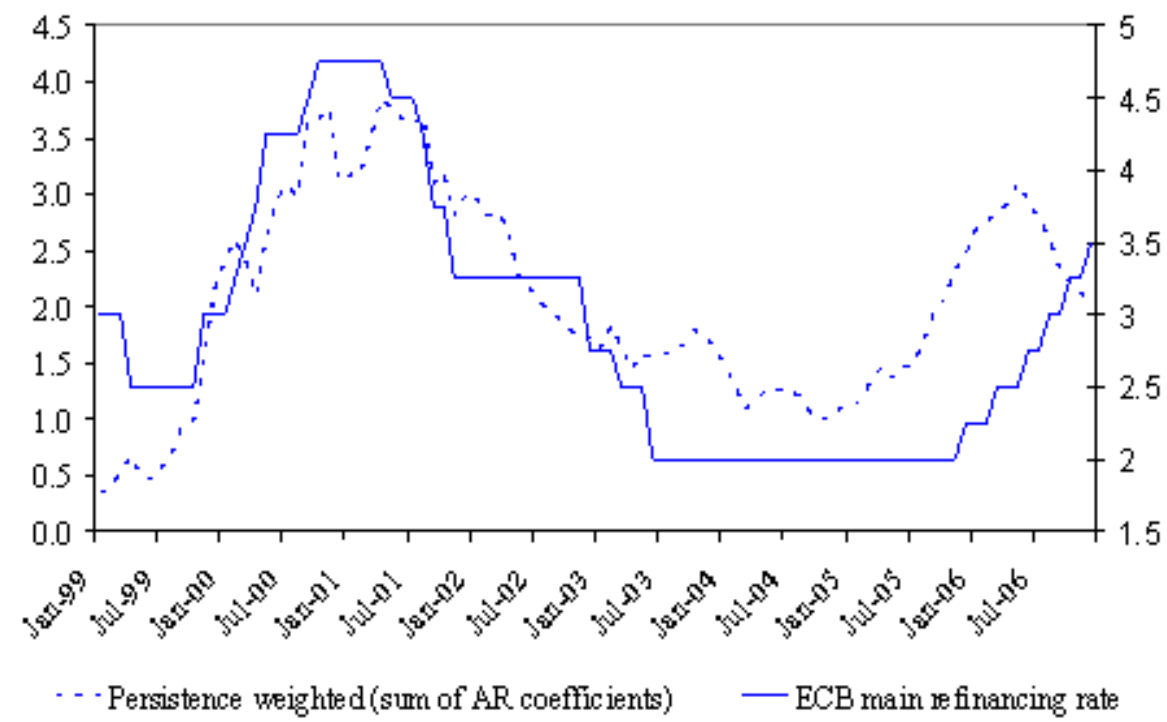

Although one has to eschew simplistic representations of ECB monetary policy decisions, it is intriguing to think that the persistence-weighted measure may contain some information on the persistent movements in prices that is reflected in medium-term inflationary pressure, to which the ECB has obviously reacted. In this respect, it should be emphasised that the correlation between the persistence-weighted measure and the ECB main refinancing rate is as high as 0.73 , while the correlation with headline HICP inflation is only 0.2 . It should be noted that we are not suggesting that the ECB has mechanistically reacted to this particular (or any other) indicator of core inflation; we are rather suggesting that our indicator of core inflation is itself correlated with the medium-term inflation outlook to which the ECB does react.

A slightly more sophisticated way to single out the correlation between the persistenceweighted indicator of core inflation and the ECB main refinancing rate is to estimate a simple Taylor-type policy rule as follows

$$
R_{t}=\bar{R}+\alpha y_{t-2}+\beta \widetilde{\pi}_{t-2}+\varepsilon_{t}
$$

where $R_{t}$ is the ECB main refinancing rate, $\bar{R}$ is a constant term, $y_{t}$ is a measure of economic slack at monthly frequency (we take industrial production detrended with the HodrickPrescott filter ${ }^{11}$ ) and $\widetilde{\pi}_{t}$ is annual core inflation rate based on the $\widetilde{\gamma}_{i T}^{A R}$ weights, minus headline HICP inflation. The basic idea behind the inclusion of the last term is that when core inflation is above (below) HICP inflation, this should signal an increase (decrease) of inflationary pressure, following for example Marques, Neves and Sarmento (2003). Therefore, a positive

\footnotetext{
${ }^{11}$ Note that we apply the one-sided HP filter in order to make it real time, but we do not use real time data proper for industrial production.
} 
and significant $\beta$ implies that the core inflation measure includes information that is relevant for monetary policy. Note that we include both core inflation and the industrial production gap with two monthly lags, in order to have a realistic picture of the information set available for the ECB decision-making body, which takes monetary policy decisions at the beginning of each month. ${ }^{12}$

The result of the estimation of the policy rule is reported in Table 2. It is quite striking to note that the persistence-weighted core inflation indicator has a positive and statistically significant coefficient while the industrial production gap is insignificant. We obtain similar results also for the second persistence-weighted measure based on the number of mean crossings (see also Figure 5). We also tried to replace the $\widetilde{\pi}_{t}$ indicator with seven alternative popular measures of core inflation in the euro area, namely (i) HICP inflation excluding energy and unprocessed food; (ii) the trimmed mean, obtained by excluding the $10 \%$ most volatile components at each point in time; (iii) the trimmed mean, obtained by excluding the $32 \%$ most volatile components at each point in time; (iv) the median increase in the price distribution underlying the HICP (corresponding to a trimmed mean where all components are excluded except one); (v) one measure based on the dynamic factor model proposed by Cristadoro et. al. (2005), "Eurocore", which exploits a database of 149 disaggregated price series; ${ }^{13}$ (vi) a second factor model series proposed by Camba-Mendez and Kapetanios (2005), available until October 2005; (vii) a variance-adjusted core inflation measure. ${ }^{14}$ Remarkably, in none of the cases was the $\beta$ coefficient positive and statistically significant, and neither was for the third persistence-weighted measures proposed in this paper (i.e. based on $\widetilde{\gamma}_{i T}^{\text {Corr }}$ weights; see Figure 6). In several cases, the $\beta$ coefficient even had a statistically significant wrong sign.

\footnotetext{
${ }^{12}$ We also tried to include headline HICP inflation separately in the equation, but this had a negative sign (in practically all specifications) and was therefore dropped from the equation.

${ }^{13}$ The program we used to compute the Eurocore index has been developed by M. Lenza.

${ }^{14}$ Note that this last measure is also computed in real time, as the persistence-weighted ones.
} 

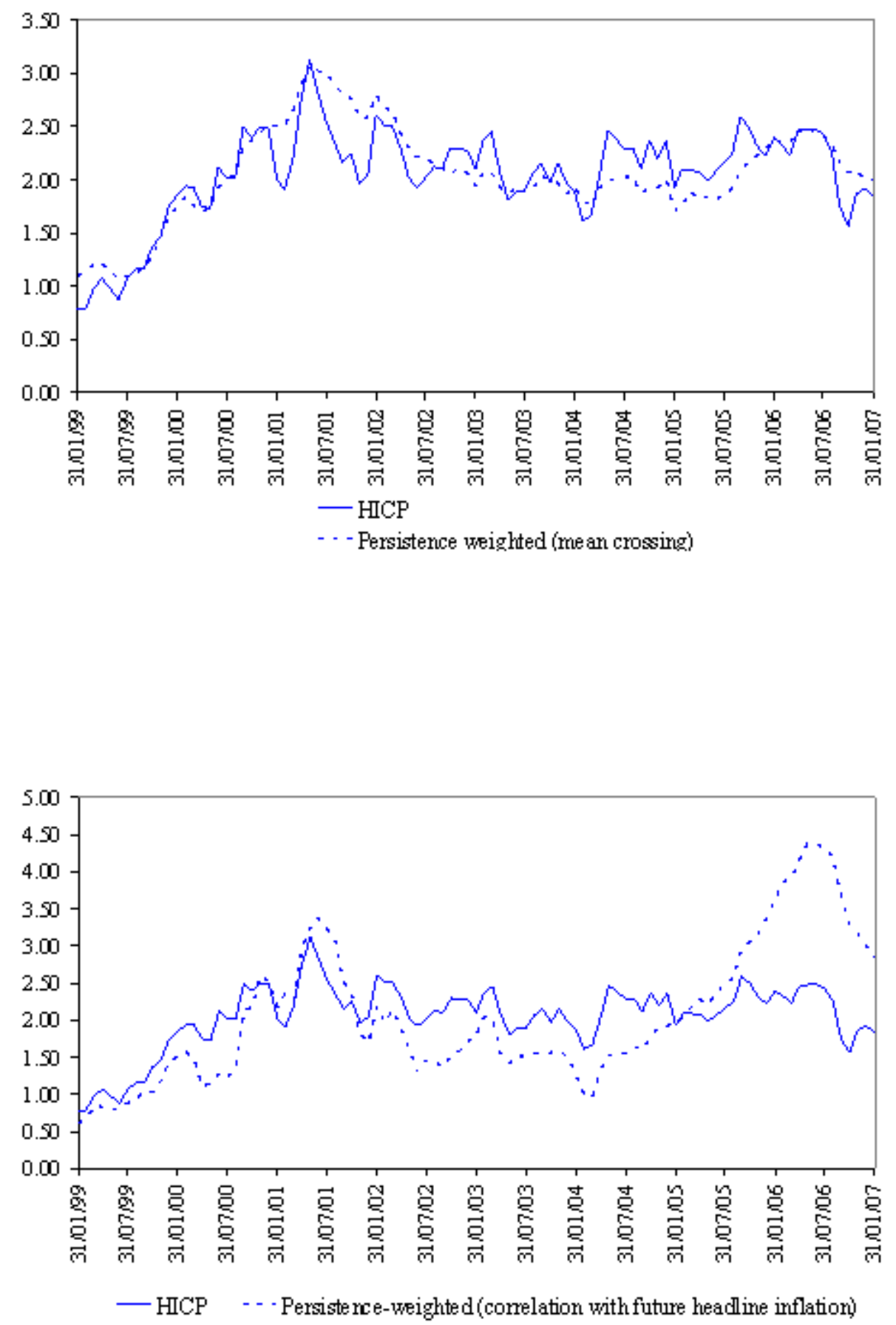
Table 2: Estimates of the policy rule in (4)

\begin{tabular}{|l|l|}
\hline $\bar{R}$ & $2.91^{* *}(0.05)$ \\
\hline$\alpha$ & $0.02(0.04)$ \\
\hline $\boldsymbol{\beta}$ & $\mathbf{1 . 0 8}^{* *}(\mathbf{0 . 0 7})$ \\
\hline Adjusted R squared & 0.72 \\
\hline DW & 0.36 \\
\hline
\end{tabular}

Note: Standard errors in parentheses; all coefficients are significant at the $5 \%$ confidence level. Sample period: January 1999 to January 2007. ** denotes statistical significance at the 1 per cent level.

\section{A closer look at the individual HICP components}

We now turn to analyse the estimated persistence of the individual HICP components. The main goal of this Section is to identify HICP components for which the persistence weight may differ significantly from the HICP (COICOP weight). Of particular interest are those components which have high persistence and may therefore contain a larger amount of information about future price trends, in contrast with components whose fluctuations are typically more short-lived.

\subsection{The weight of the complete set of 93 components}

Looking at the complete set of the 93 components of the HICP, Figure 7 displays a scatter plot of the persistence weights $\widetilde{\gamma}_{i T}^{A R}$ and the original HICP weights, as average over the whole sample period (from January 1998 to January 2007). It is quite striking to note that the two sets of weights are practically orthogonal (the R-squared is less than .02): there is no relationship whatsoever between persistence weights and HICP weights. That is also true for the other two variants considered in this paper; for example, Figure 8 reports the scatter plot with the HICP weights vis-a-vis the weights obtained with the mean crossing measure, $\widetilde{\gamma}_{i T}^{M C}$.

By contrast, there is a positive and significant correlation between the weights of the three different persistence weights. For instance, Figure 9 reports the scatter plot with the $\widetilde{\gamma}_{i T}^{A R}$ and $\widetilde{\gamma}_{i T}^{M C}$ weights. The R-squared is above 0.3 and the curve is upward sloped.

\subsection{What are the most persistent components of the HICP? Some surprises}

Table 3 reports some key sub-components of the HICP, as reported in Table 1 above, and their average COICOP and persistence $\left(\widetilde{\gamma}_{i T}^{A R}\right)$ weights during the sample period under consideration (January 1998 to January 2007). A couple of large discrepancies between the 


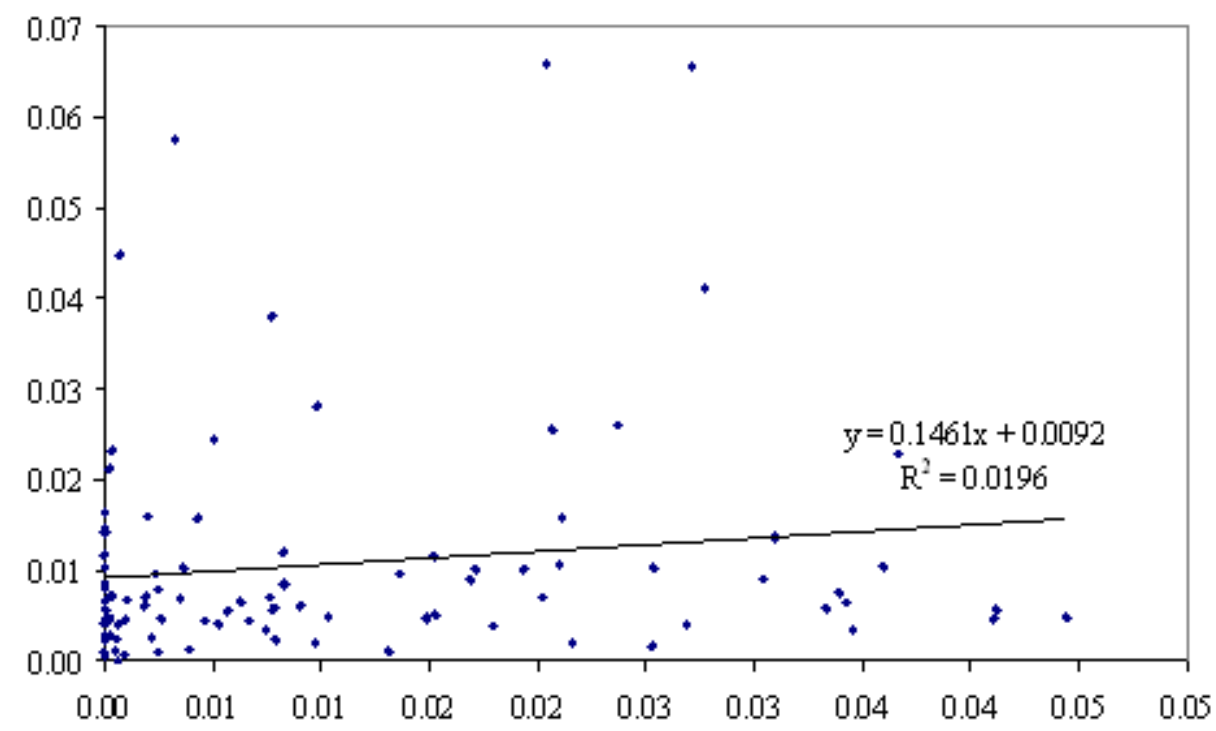

Figure 4: Average persistence (sum of AR) and HICP weights.

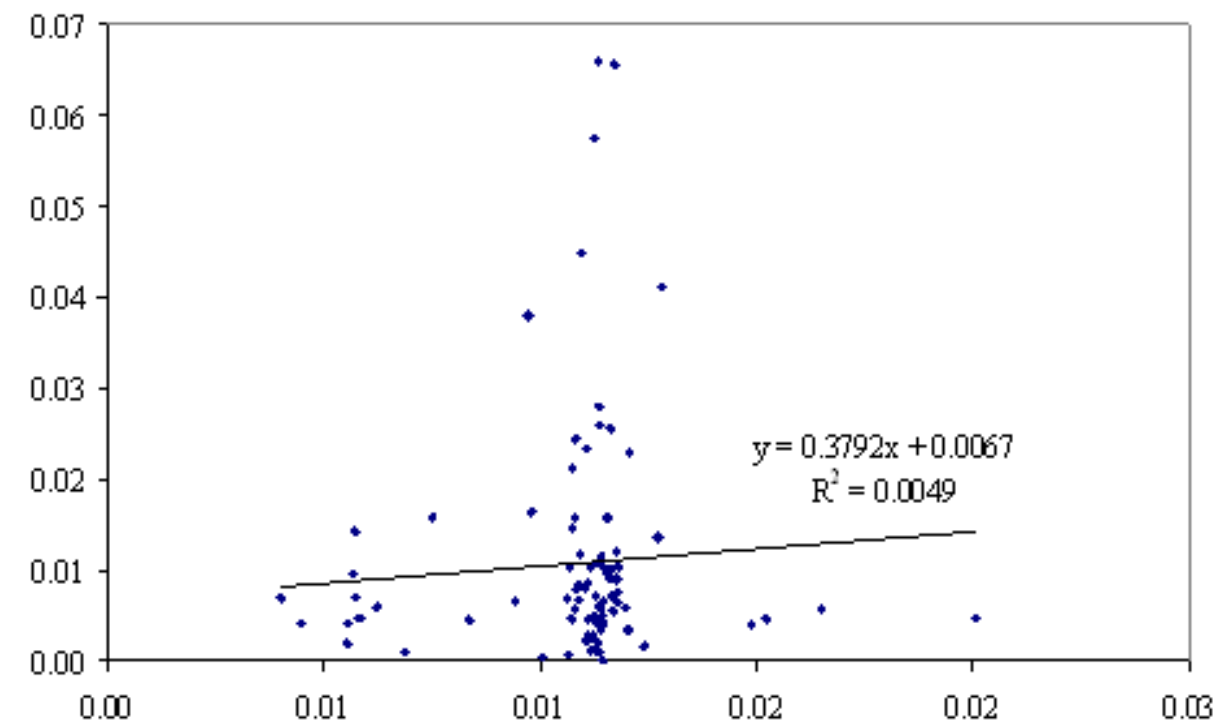

Figure 5: Average persistence (mean crossing) and HICP weights. 


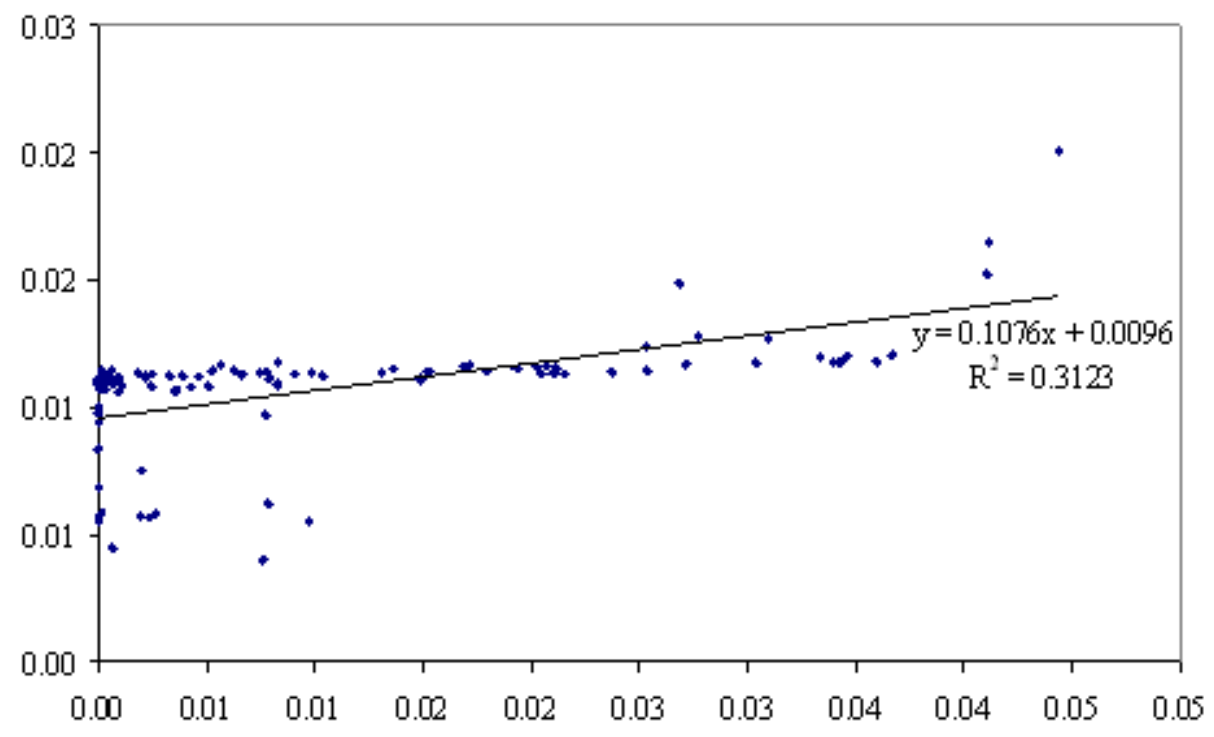

Figure 6: Average of persistence weights (sum of AR and mean crossing).

persistence and COICOP weights stand out. Given the persistent rise in oil price inflation during a large part of the sample period, one would have expected the persistence weight of energy to be much larger than the HICP weight; in fact, it is larger, but not very significantly so. It is unprocessed food the sub-component for which the persistence weight is largest compared with the HICP weight. Together, the persistence weight of energy and unprocessed food exceeds the HICP weights by 14 percentage points. Moreover, non-energy industrial goods are also over-represented in the persistence weighted index compared with the HICP.

The reduced weight on services prices is the mirror image of the larger weight on energy, unprocessed food and non-energy industrial goods. The most under-represented component of services is "Restaurant and cafés", despite the fact that this sector has been allegedly hit by the permanent shock of the 2002 cash changeover. One way to interpret this result is to think not only in terms of frequency of price adjustment as is typically studied in the literature (see ECB 2005), but also in terms of underlying shocks hitting the fundamental determinants of prices (marginal costs) in individual sectors. Over recent years, the shocks which have hit commodity and manufacturing prices (some of which, arguably, of a global nature) may have been more persistent than shocks (arguably of a more local nature) hitting services prices. The advantage of a measure such as the one computed in this paper is precisely that of being agnostic about the persistence of certain price components and let the data speak, especially when (which is most often the case) the underlying shock hitting prices is not observable. A measure that permanently excludes some of the price components cannot take into account changes in the persistence of the individual price series which reflect the 
nature of the shocks hitting the economy. ${ }^{15}$ The fact that the persistence weighted measure better captures the nature of the shocks appears to be corroborated by its strong correlation with the ECB monetary policy decisions during the considered period.

Some of these findings may be challenging previous evidence on the euro area. Altissimo et. al. (2006) found that changes in energy and unprocessed food prices were the less persistent, while changes in processed food, non-energy industrial goods and service prices appeared to be more persistent. One important factor behind this difference is certainly the sample period. While our study is based on the last 12 years, the evidence in Altissimo et. al. (2006) relates to a much longer sample period. The recent past has been characterised, for example, by a more persistent than usual oil price shocks which can explain the high persistence we find for energy components. This leads us to emphasise that persistence appears to be a time-varying feature, not only for the inflation process as a whole, but also for the individual sub-components of the HICP (or any other consumer price index).

Related to this matter, it is also interesting to look at the stability of the persistence weights. This is very important, indeed crucial for the useful of any measure of persistenceweighted core inflation: there must be some continuity in the persistence measure, for the weights to be informative about future developments. It turns out that - apart perhaps from processed food - the weights of the main components of the HICP have been not too unstable within our sample period, as reported in Figure 10. Some important components of the services sub-aggregate are reported in Figure 11. In this case, the weights of these more dis-aggregated components appear to be less stable, probably reflecting some idiosyncratic errors in the estimation of the persistence of the process.

\footnotetext{
${ }^{15}$ Ideally, therefore, a persistence weighted measure should be based on persistence estimates that are timevarying. As mentioned earlier, we only apply a recursive estimation in this paper due to data limitations.
} 


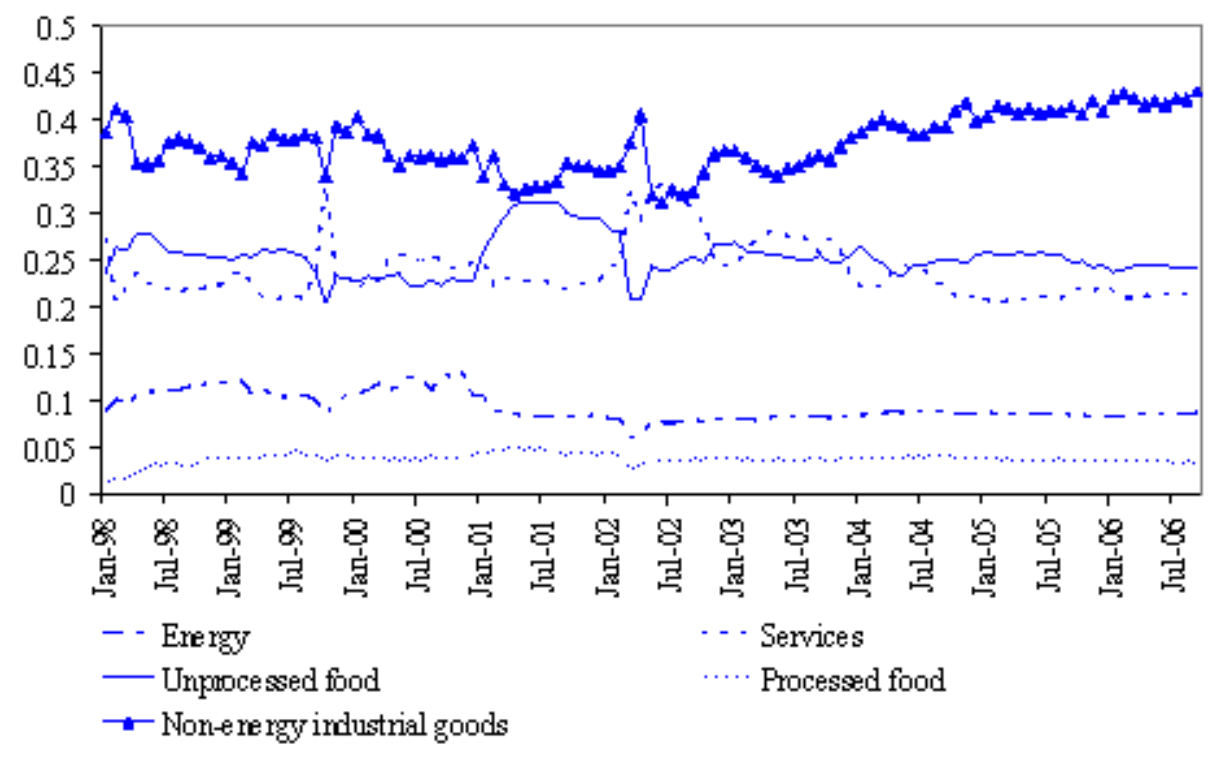

Figure 7: Time series for persistence weights (sum of AR).

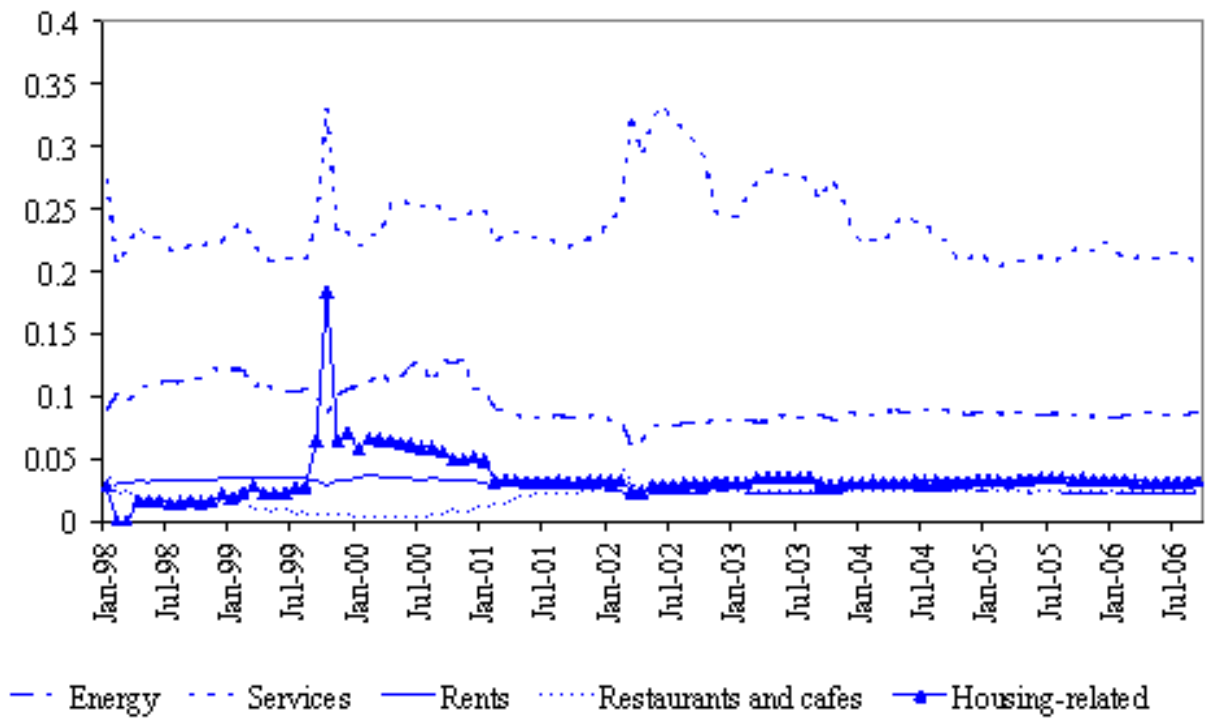

Figure 8: Time series for persistence weights (sum of AR), services prices. 
Table 3: Average weights in the baseline persistence-weighted index and in the HICP

\begin{tabular}{||c||l||l|l|}
\hline \hline Item & HICP & $\widetilde{\gamma}_{i T}^{A R}$ & $\widetilde{\gamma}_{i T}^{A R}-$ HICP \\
\hline \hline Food (incl. alcohol and tobacco) & 20.2 & 29.2 & 9.0 \\
\hline o/w processed & 8.0 & 3.8 & -4.2 \\
\hline o/w unprocessed & 12.2 & 25.4 & 13.1 \\
\hline Industrial goods & 40.5 & 47.1 & 6.5 \\
\hline o/w non-energy & 31.9 & 37.7 & 5.8 \\
\hline o/w energy & 8.6 & 9.3 & 0.7 \\
\hline Services & 39.2 & 23.8 & -15.4 \\
\hline o/w rents paid by tenants & 6.5 & 3.1 & -3.4 \\
\hline o/w restaurants and cafes & 6.6 & 2.1 & -4.5 \\
\hline o/w housing & 3.8 & 3.7 & -0.1 \\
\hline o/w transport & 5.7 & 4.4 & -1.3 \\
\hline
\end{tabular}

Note: Averages are taken over the sample period from January 1998 to December 2006. Data are in percentage points.

\section{Conclusions}

In this paper we have built three alternative measures of core inflation in the euro area and we have analysed their properties in terms of correlation with current and future headline HICP inflation and with the ECB's monetary policy decisions. We have argued, in line with some recent literature, that in an environment of price stability in which the central bank reacts aggressively and in a timely manner to inflationary pressure it is highly unlikely to find core inflation (or any other) indicators that may be systematically correlated with future headline inflation. More revealing about the potential usefulness of a core inflation indicator should be its correlation with the monetary policy decisions. We have shown that our baseline measure of persistence-weighted core inflation scores remarkably well in this respect. However, it goes without saying that, given the wide information set taken into consideration by the ECB, it is highly unlikely that any core inflation indicator can be a "sufficient statistic" to explain monetary policy decisions in the euro area.

Another interesting result of this study is that there appears to be significant time variation in the persistence of the sub-components of the euro area HICP. In contrast with the findings of the Inflation Persistence Network, we find services prices to be under-weighted in our persistence-weighted indicator, while energy and food prices are over-weighted compared with the original HICP weights. This reflects, in our view, the different nature of the shocks which have predominantly hit prices in the sample period we use in this paper (from 1995 onwards) compared with the longer sample period studied in previous work. 
In terms of avenues for future research, our results are encouraging for following up this work on countries where disaggregated CPI data are available for a longer sample period. The United States, in particular, appears to be a suitable candidate for an application of this approach, which could also usefully incorporate elements of time variation in the parameters governing persistence. 


\section{References}

[1] Andrews D.W.K. and Chen H.-Y. (1994), "Approximately median-unbiased estimation of autoregressive models ", Journal of Business and Economic Statistics 12(2),187-204.

[2] Altissimo F. and Corradi V. (2003), "Strong rules for detecting the number of breaks in a time series ", Journal of Econometrics 117, 207-244.

[3] Altissimo, F., L. Bilke, A. Levin, T. Mathä, and B. Mojon (2006), "Sectoral and aggregate inflation dynamics in the euro area", Journal of the European Economic Association, 4(2-3), 585-593, April-May 2006.

[4] Bilke, L. (2005), "Break in the mean and persistence of inflation: a sectoral analysis of French CPI ", ECB Working Paper Series, 463, March 2005.

[5] Blinder, A., (1997), "Commentary", Review, Federal Reserve Bank of Saint Louis, May-June 1997.

[6] Camba-Mendez, G. and G. Kapetanios (2005): "Forecasting euro area inflation using dynamic factor measures of underlying inflation", Journal of Forecasting, 24, pp. 491503.

[7] Corvoisier, S., and B. Mojon (2005), "Breaks in the mean of inflation: how they happen and what to do wth them?", ECB Working Paper Series, 451, March 2005.

[8] Cristadoro, R., Forni, M., Reichlin, L. and G. Veronese (2005): "A core inflation indicator for the euro area", Journal of Money, Credit and Banking, 37, 3, pp. 539-60.

[9] Cutler, J. (2001): "A new measure of core inflation in the UK", MPC Unit Discussion Paper n. 3.

[10] Demarco, A. (2004), "A new measure of core inflation for Malta", Central Bank of Malta Quarterly Review, 2004:2.

[11] Dias, D. and C. Robalo Marques (2005): "Using mean reversion as a measure of persistence", ECB Working Paper Series n. 450.

[12] European Central Bank (2001): Measures of underlying inflation in the euro area, Monthly Bulletin, July.

[13] European Central Bank (2005): Price setting behaviour in the euro area, Monthly Bulletin, November.

[14] Mankikar, A. and J. Paisley (2004): "Core inflation: a critical guide", Bank of England Working Paper n. 242. 
[15] Perron P. (1990), "Testing for a unit root in a time series with a changing mean", Journal of Business and Economic Statistics, 8, 153-162.

[16] Pedersen M. (2005), "An alternative measure of core inflation", Danmarks Nationalbank Working Papers, n. 2005-33, December.

[17] Marques, C. R., P. D. Neves, and L. M. Sarmento (2003), "Evaluating core inflation indicators", Economic modelling, 20 (2003), 765-775.

[18] Roger, S. (1998): "Core inflation: concepts, uses and measurement", Reserve Bank of New Zealand Discussion Paper, 98/9.

[19] Vega, J. L. and M. Wynne (2003): "An evaluation of some measures of core inflation for the euro area", German Economic Review, 4, 3, pp 269-306.

[20] Wynne, M. A. (1999): "Core inflation: a review of some conceptual issues", Working Paper of the Federal Reserve Bank of Dallas, 99-03. 


\section{European Central Bank Working Paper Series}

For a complete list of Working Papers published by the ECB, please visit the ECB's website (http://www.ecb.europa.eu).

877 "What are the effects of fiscal policy shocks? A VAR-based comparative analysis" by D. Caldara and C. Kamps, March 2008.

878 "Nominal and real interest rates during an optimal disinflation in New Keynesian models" by M. Hagedorn, March 2008.

879 "Government risk premiums in the bond market: EMU and Canada" by L. Schuknecht, J. von Hagen and G. Wolswijk, March 2008.

880 "On policy interactions among nations: when do cooperation and commitment matter?" by H. Kempf and L. von Thadden, March 2008.

88I "Imperfect predictability and mutual fund dynamics: how managers use predictors in changing systematic risk" by G. Amisano and R. Savona, March 2008.

882 "Forecasting world trade: direct versus "bottom-up" approaches" by M. Burgert and S. Dées, March 2008.

883 "Assessing the benefits of international portfolio diversification in bonds and stocks" by R. A. De Santis and L. Sarno, March 2008.

884 "A quantitative perspective on optimal monetary policy cooperation between the US and the euro area" by S. Adjemian, M. Darracq Pariès and F. Smets, March 2008.

885 "Impact of bank competition on the interest rate pass-through in the euro area" by M. van Leuvensteijn, C. Kok Sørensen, J. A. Bikker and A. A. R. J. M. van Rixtel, March 2008.

886 "International evidence on sticky consumption growth" by C. D. Carroll, J. Slacalek and M. Sommer, March 2008.

887 "Labor supply after transition: evidence from the Czech Republic" by A. Bičáková, J. Slacalek and M. Slavík, March 2008.

888 "House prices, money, credit and the macroeconomy" by C. Goodhart and B. Hofmann, April 2008.

889 "Credit and the natural rate of interest" by F. De Fiore and O. Tristani, April 2008.

890 "Globalisation, domestic inflation and global output gaps: evidence from the euro area" by A. Calza, April 2008.

891 "House prices and the stance of monetary policy" by M. Jarociński and F. Smets, April 2008.

892 "Identification of New Keynesian Phillips Curves from a global perspective" by S. Dées, M. H. Pesaran, L. V. Smith and R. P. Smith, April 2008.

893 "Sticky wages: evidence from quarterly microeconomic data" by T. Heckel, H. Le Bihan and M. Montornès, May 2008.

894 "The role of country-specific trade and survey data in forecasting euro area manufacturing production: perspective from large panel factor models" by M. Darracq Pariès and L. Maurin, May 2008.

895 "On the empirical evidence of the intertemporal current account model for the euro area countries" by M. Ca'Zorzi and M. Rubaszek, May 2008. 
896 "The Maastricht convergence criteria and optimal monetary policy for the EMU accession countries" by A. Lipińska, May 2008.

897 “DSGE-modelling when agents are imperfectly informed” by P. De Grauwe, May 2008.

898 "Central bank communication and monetary policy: a survey of theory and evidence" by A. S. Blinder, M. Ehrmann, M. Fratzscher, J. De Haan and D.-J. Jansen, May 2008.

899 "Robust monetary rules under unstructured and structured model uncertainty" by P. Levine and J. Pearlman, May 2008.

900 "Forecasting inflation and tracking monetary policy in the euro area: does national information help?" by R. Cristadoro, F. Venditti and G. Saporito, May 2008.

901 "The usefulness of infra-annual government cash budgetary data for fiscal forecasting in the euro area" by L. Onorante, D. J. Pedregal, J. J. Pérez and S. Signorini, May 2008.

902 "Fiscal consolidation in the euro area: long-run benefits and short-run costs" by G. Coenen, M. Mohr and R. Straub, May 2008.

903 "A robust criterion for determining the number of static factors in approximate factor models" by L. Alessi, M. Barigozzi and M. Capasso, May 2008.

904 "Does money matter in the IS curve? The case of the UK" by B. E. Jones and L. Stracca, June 2008.

905 "A persistence-weighted measure of core inflation in the euro area" by L. Bilke and L. Stracca, June 2008. 


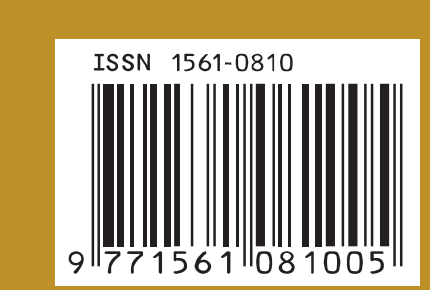

